\title{
Oportunidades de la pandemia en la educación musical universitaria: motivación para acciones académicas sustentables
}

Irma Susana Carbajal Vaca

\section{Resumen}

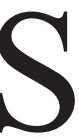
e analizaron acciones académicas realizadas en la Licenciatura en Música de la Universidad Autónoma de Aguascalientes (UAA) para dar respuesta a las demandas educativas provocadas por la situación de confinamiento iniciada en marzo de 2020. Los mecanismos motivacionales y volitivos que impulsaron las acciones fueron contrastados teóricamente e interpretados para este trabajo como oportunidades de la pandemia CoviD-19, para la educación universitaria. El resultado puede considerarse un indicador hacia un cambio social que va encaminado a prácticas educativas más sostenibles.

Palabras clave: educación musical universitaria, Covid-19, cambio social, educación en línea, sustentabilidad educativa.

\section{Introducción}

A inicios de 2021, desde la perspectiva de la historia del tiempo presente, quien suscribe este texto, publicó un artículo que capturó una primera imagen de la recepción del Programa Emergente de Educación a Distancia (PEED) de los estudiantes y profesores de la Licenciatura en Música de la Universidad Autónoma de Aguascalientes (UAA), respecto a la situación de confinamiento iniciado el 14 de marzo de 2020. Las respuestas al cuestionario, aplicado entre mayo y junio de ese año, mostraron algunos de los cambios en la dinámica de trabajo, los cuales podrían ser mantenidos en el currículo de la Licenciatura en Música, ya que, aun sin haber sido un propósito explícito, atendían problemáticas de movilidad y espacio previas al confinamiento. En ese primer momento, en un ambiente de solidaridad y respeto, tanto estudiantes como profesores mostraron flexibilidad y disposición al cambio. Se señalaron como acciones necesarias, la implementación de cursos de capacitación para profesores y estudiantes en los que se incluyera el uso de tecnología y temáticas para comprender el estudio autónomo, el desarrollo de actitu- des como la paciencia, el trabajo en equipo, la autocrítica y la comunicación (Carbajal Vaca, 2021).

A un año de distancia de la aplicación del cuestionario, en este texto se muestran algunas acciones emprendidas por la docente para responder a las demandas académicas propiciadas por la situación de confinamiento. Se analizan los mecanismos motivacionales y volicionales que impulsaron las distintas acciones académicas de acuerdo con teorías de la motivación y de la acción (Bartels et al., 2009; Heckhausen y Heckhausen, 2018), las cuales se interpretaron como oportunidades de la pandemia Covid-19 (Leal Filho, 2021; Spinney, 2021) en la formación musical universitaria.

\section{Motivación para la acción}

Heckhausen y Heckhausen (2018) sostienen que el comportamiento humano está orientado a efectuar cambios en el medio ambiente; ésa es la razón para perseguir o no una meta. Este comportamiento se fundamenta en dos características universales de la acción humana: 1) la lucha por 
el control y 2) la organización para asumir el compromiso o liberarse de él. Estos autores destacan la existencia suficiente de evidencias teóricas y empíricas para aceptar que los mamíferos desarrollan mecanismos asociados a la lucha por el control, como son la preferencia por los comportamientos que producen contingencias; es decir, hacia eventos posibles pero sobre los cuales no se tiene certeza; la exploración como sistema motivacional universal para extender el rango de control del entorno; y la asimetría en las respuestas afectivas a eventos positivos y negativos, razón del sujeto para aspirar siempre a nuevas metas en lugar de conformarse con los éxitos.

La motivación para aspirar a una meta está influenciada por factores personales y factores de situación, incluidos los resultados anticipados de las acciones y sus consecuencias. Estos factores no pueden disociarse, pues una persona siempre se encuentra inmersa en una situación de manera particular. Entre los factores personales están: los comportamientos universales, expresados en las necesidades físicas básicas; los motivos implícitos que responden a características propias de cada individuo; y los motivos explícitos actualizados en las metas individuales.

Por su parte, los factores de situación se refieren a las oportunidades e incentivos: resultados positivos o negativos de una circunstancia, probablemente asociados con la acción, con su resultado, o bien con varias consecuencias de la acción, mismas que están involucradas con regulaciones motivacionales y volicionales.

La volición se diferencia de la motivación en lo siguiente: si bien la motivación es necesaria para establecer metas, la volición evita su abandono porque es la encargada de mantener la perseverancia cuando se presentan otras metas en competencia (Bartels et al., 2009).

\section{Pandemia y sustentabilidad}

Trabajos recientes, como los compilados por Walter Leal Filho (2021) en el texto "CoviD-19: Allanando el camino por un mundo más sostenible", señalan que a pesar del impacto severo de la pandemia en la docencia e investigación, es posible abrir nuevas oportunidades para la sustentabilidad. Acciones concretas como la restricción en la movilidad, con lo cual se han reducido el consumo de combustibles y los costos de traslado, así como el aumento de estrategias de aprendizaje en línea son muestra de ello. Leal Filho, convencido de la necesidad de investigar con mayor profundidad para prepararnos a escenarios de futuras pandemias, propone aprovechar estas expe- riencias como una oportunidad para realizar un cambio global dirigido hacia una mayor sostenibilidad ambiental, económica y social.

Coincidentemente, el investigador Justin Spinney (2021) considera la muerte de la movilidad como una oportunidad para priorizar la sostenibilidad y reorganizar las ciudades para reducir las desigualdades en salud, lo cual mitigaría el riesgo de pandemias futuras y contrarrestaría el desequilibrio climático causado por este exceso. El autor señala la necesidad de generar una agenda con la meta de comprender las diferencias en cuanto a los cambios en la movilidad y las prácticas laborales para que éstas no favorezcan sólo a quienes pueden adaptarse a los nuevos estilos de vida, como el trabajo desde casa.

En junio de 2021 se realizó la segunda emisión virtual del Coloquio de Educación Musical a Nivel Superior (CIEMNS). Entre los comentarios de los participantes llama la atención uno coincidente con las apreciaciones de Leal Filho y de Spinney, el cual, como respuesta a los esfuerzos de la comunidad académica del Departamento de Música ante el confinamiento, se percibe motivante y esperanzador: "[...] los investigadores y docentes han transformado la pandemia en una tierra de oportunidades" (UAA, 2021a). Ante estos resultados, la autora se pregunta sobre los mecanismos motivacionales y volicionales para la acción a la luz de los factores personales y de situación experimentados en la tarea docente en el contexto de un evento de contingencia como el de la pandemia.

\section{Oportunidades en confinamiento}

Cada año, los profesores investigadores tenemos la posibilidad de registrar un proyecto al cual la UAA asigna un presupuesto para su realización (UAA, 2021b). En sintonía con la situación mundial, la UAA suspendió la movilidad, lo cual abrió nuevas oportunidades para prácticas más sustentables y convenientes para la investigación en 2020 y 2021, debido a su realización en modalidad virtual. Para el presupuesto destinado al traslado, consumo de alimentos y hospedaje de investigadores para asistir a reuniones académicas y trabajo de campo, se planearon otras actividades, como gestionar publicaciones, apoyar a instructores beca (UAA, 2012) y fortalecer los equipos de investigación con la contratación de técnicos de apoyo (UAA, 2006).

Estos cambios impactaron en una de las tareas más apremiantes para los cuerpos académicos: generar cursos de capacitación y actualización para la comu- 
nidad académica. En 2020 el maestro Marco Antonio Pruneda Tavárez ofreció el curso-taller 'Herramientas de transmisión en línea' a los organizadores y colaboradores del CIEMNS. Asimismo, la entonces maestranda Karla Jacqueline Silva Doray Ledezma impartió el taller de "Producción radiofónica desde casa", para capacitar a los colaboradores de producción del programa "Ventana al Sonido" coordinado por el cuerpo académico UAA-CA-117. Durante el semestre enero-junio de 2021, ya como egresada de la Maestría en Arte y en su calidad de técnico de investigación, Silva Doray Ledezma impartió por segunda ocasión el curso-taller de producción radiofónica en línea para vigorizar las emisiones del programa. Asimismo, gracias a la interinstitucionalización alcanzada a distancia, para Brenda Araceli Galindo de la Llave, maestranda de la Universidad Nacional Autónoma de México (UNAM), fue posible impartir en movilidad virtual en la UAA, un curso-taller de profesionalización en conciertos didácticos. Mediante estos talleres se fortaleció la vinculación institucional y la internacionalización, ya que participaron estudiantes y profesores de instituciones nacionales como el Conservatorio Nacional de Música (CNM), de la Facultad de Música de la unam, de la Universidad de Ciencias y Artes de Chiapas (UNICACH), de la Universidad Autónoma de Coahuila (UAC), de la Universidad Autónoma del Estado de Hidalgo (UAEH), de la Universidad Autónoma de Yucatán (UADY), de la Benemérita Universidad Autónoma de Puebla (BUAP), del Tec Milenio, así como de otras instituciones en Argentina, Colombia, Ecuador y Guatemala.

Las actividades en línea han permitido una mayor presencia de la autora en espacios nacionales e internacionales, como la Universidad Autónoma Metropolitana, el Centro Nacional de Investigación, Documentación e Información Musical (CENIDIM), la Facultad de Música de la UNAM, la Universidad Nacional de Cuyo, Argentina (UNCUYO), la Universidad Alberto Hurtado en Chile, la Asociación Argentina de Performance Musical (АArpem). Asimismo, en este tiempo ha sido posible la colaboración cercana con proyectos de otros países, como fue el caso de la investigación "Percepción de la educación musical del siglo XXI: un acercamiento fenomenológico a las visiones de docentes universitarios latinoamericanos" (Carbajal Vaca y Saez, 2020), ${ }^{1}$ realizada con la doctora Alejandra Saez de la UnCUYO.

1 Conferencia transmitida en línea por la Asociación Argentina de Performance Musical. "Educando al músico del siglo xxi". Disponible en https://www.youtube.com/watch?v=zRuKmu8y_ As\&t=36s
En los CIEMNS 2020 y 2021 y las emisiones del Seminario Permanente (SEMPER) realizados en línea, se presentó un incremento significativo en la participación internacional gracias a la simplificación en la gestión por costos de transporte y viáticos, pero también por el apoyo institucional que nos ha permitido abrir nuestros espacios educativos. También fue posible la vinculación con proyectos de egresados de la UAA, involucrados en acciones académicas en sus espacios laborales, como fue el caso del licenciado Jesús Andrés García Santín, quien organizó el primer Simposio de Mujeres Cornistas.

\section{Conclusiones}

En este análisis se valoran como aciertos: la reasignación de los presupuestos para incentivar la formación de investigadores, la integración de instructores beca en los proyectos de investigación para apoyar a los estudiantes afectados por la pandemia, la inclusión de profesores y estudiantes de posgrado en los proyectos de capacitación y actualización docente local, nacional e internacional emprendidos por el Cuerpo Académico UAA-CA-117 y la documentación de experiencias encaminadas a la reflexión sobre futuros escenarios de pandemia y desequilibrios climáticos que, de acuerdo con los estudios recientes revisados para este trabajo, han sido resultado de prácticas inadecuadas, como el exceso de movilidad.

Esta exploración parece corroborar las características universales del comportamiento humano, puesto que se reconocen mecanismos motivacionales y volicionales para luchar por el control de la contingencia (Heckhausen y Heckhausen, 2018). Algunas de las acciones fueron influidas por factores personales derivados del interés de investigación de la autora por comprender el cambio social en el currículo de las licenciaturas en Música del país (Carbajal Vaca, 2020), y por factores de la situación de confinamiento tales como: la necesidad de capacitación, el reconocimiento del valor del trabajo en equipo, la consolidación del sentido de comunidad, entre otros.

Aun en el halo de incertidumbre e inexperiencia compartido por los docentes en marzo de 2020, asumimos el reto de explorar posibilidades para continuar con los compromisos académicos previos, como el programa de radio, el CIEMNS y el SEMPER. Esto ratifica la preferencia por los comportamientos generadores de contingencias, como lo señala la teoría.

Se evidencia la respuesta oportuna de la UAA a las necesidades globales, y esta documentación contribuye a 
la reflexión universitaria para comprender las nuevas prácticas laborales, como el trabajo desde casa, el cual ha sido posible y exitoso en todas las acciones de docencia, investigación, gestión y vinculación que le competen a la autora.

En síntesis, las oportunidades abiertas por la pandemia son: 1) la optimización de recursos de investigación, 2) el incremento en la internacionalización, 3) el fortalecimiento de la vinculación institucional, 4) la conformación de equipos de apoyo a la investigación y 5) la contribución en la capacitación y desarrollo de habilidades de comunicación.

\section{Fuentes de consulta}

Bartels, J. M., Magun-Jackson, S. y Kemp, A. D. (2009). La regulación volicional y la autorregulación del aprendizaje: un estudio de las diferencias individuales en la motivación de logro aproximación-evitación. Electronic journal of research in educational psychology, 7(2), 2009, 605-626. Recuperado de https://bit.ly/3aS71xT

Carbajal Vaca, I. S. (2021). Nuevas sonoridades en la educación musical: Voces universitarias ante la pandemia COVID-19. MAGOTZI Boletín Cientifico de Artes del IA, 9(17), 17-25. Recuperado de https:// doi.org/10.29057/ia.v9i17.6188

Carbajal Vaca, I. S. (2020). Implicaciones teórico-metodológicas en la historia presente de la educación musical de nivel superior en México. Revista Iberoamericana de Educación Superior, 11(32), 133147. Recuperado de https://bit.ly/3vss0kh

Carbajal Vaca, I. S. y Saez, A. (2020). Percepción de la educación musical del siglo xxI: un acercamiento fenomenológico a las visiones de docentes universitarios latinoamericanos. Cuadernos de Análisis y Debate sobre Músicas Latinoamericanas Contemporáneas 3, 156-175. Instituto Nacional de Musicología Carlos Vega. Recuperado de https://bit. ly/3AZpQcQ

Heckhausen, J. y Heckhausen, H. (Ed.). (2018). Motivation and Action. Springer.

Leal Filho, W. (Ed.). (2021). CovID-19: Paving the Way for a More Sustainable World. Springer.

Spinney, J. (2021). Mobility Is Dead: Post-pandemic Planning as an Opportunity to Prioritize Sustainability and Accessibility. Andrews, G. J., Crooks, V. A., Pearce, J. R. y Messina, J. P. (Eds). CovID-19 and Similar Futures. Pandemic Geographies. Springer.

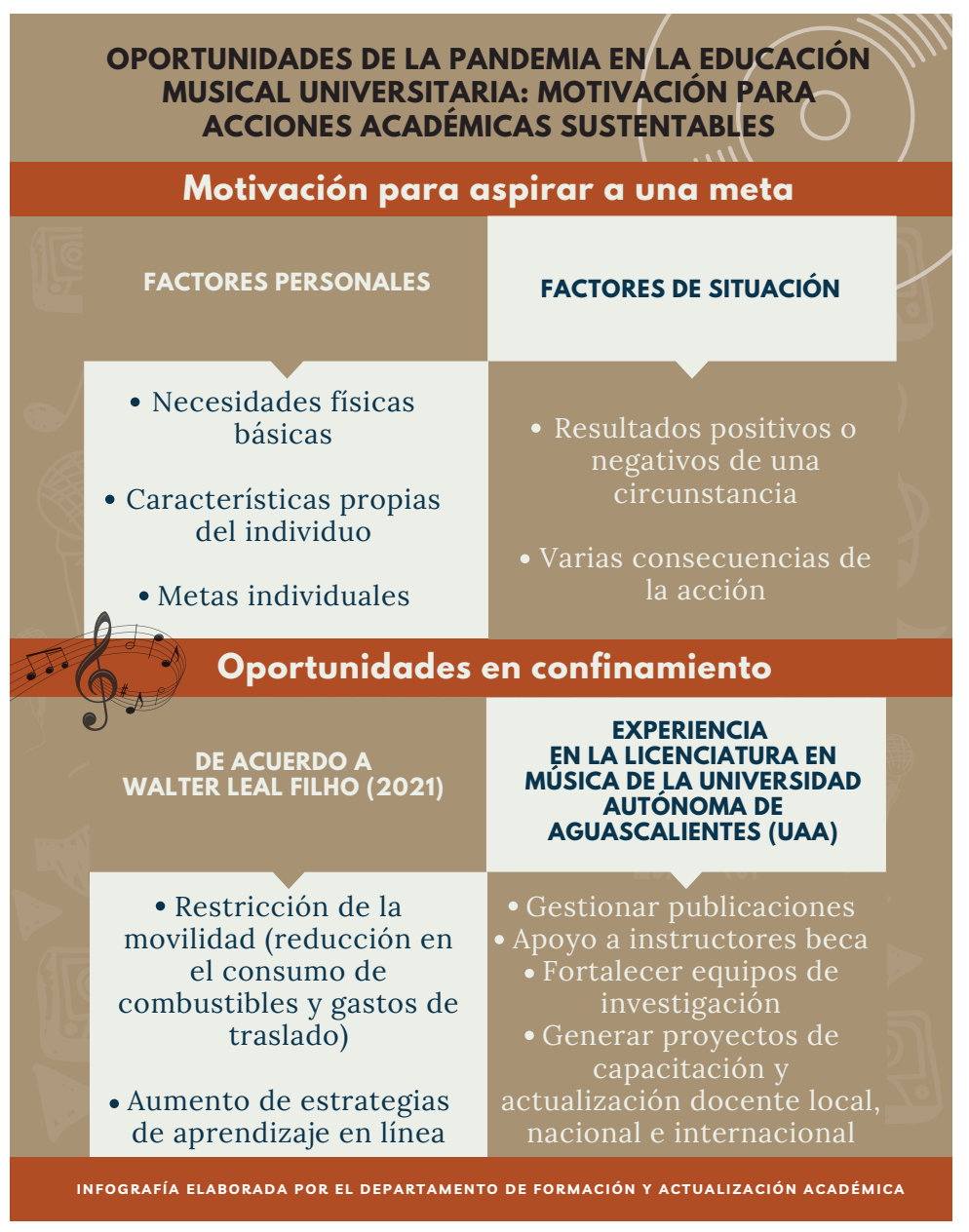

Universidad Autónoma de Aguascalientes [UAA]. (2006). Reglamento de Investigación de la UAA. Legislación Universitaria. En Correo Universitario, sexta época, núm. 12, publicado el 15 de diciembre de 2006. UAA-DGIP. Recuperado de https://bit. ly/2Xt0162

UAA. (2012). Políticas para la asignación de instructores beca, UAA-DGF. [Página web]. Recuperado de https://www.uaa.mx/direcciones/dgf/conocenos. php

UAA. (2021a). Evaluación del $8^{\circ}$ Coloquio Nacional, $5^{\circ}$ Internacional de Educación Musical a Nivel Superior vía Microsoft Forms. Archivo del Cuerpo Académico. UAA-CAC-117. Universidad Autónoma de Aguascalientes.

UAA. (2021b). Investigación Científica. [Página web]. Universidad Autónoma de Aguascalientes. Recuperado de https://bit.ly/3pnC258 